\title{
Authors Index to Abstracts
}

Aarskog D, 110

Abati L, 125

Acquafredda A, 102

Adams J, 57

Adlard P, 60, 158

Aguercif M, 148

Ahonen, 157

Ajgaonkar A, 93

Akerblom $\mathrm{H}, 49$

Albag Y, 51

Albertsson-Wik, 68

Albisu M, 2

Amand $\mathrm{Y}, 41$

Amendt P, 164

Anapliotou M, 159

Andre J, 132

Antoniazzi F, 109, 179

Arai K, 91

Arand J, 114

Arasimovicz E, 55

Argemi J, 108

Argente $\mathrm{J}, 63$

Armanini D. 152

Armitage M, 166

Arner P. 16

Arrigo T, 101

Ascione A, 23

Asensio A, 9

Asensio M, 28

Atares M, 108, 143

Attanasio A, 129, 146

Aubert M, 46, 121

Audi L, 126

Auvinet M. 132

Auzerie J, 74

Aynsley-Green, 17

Baldoni A, 162

Balestrazzi A, 69

Balks H, 156

Ballabriga A, 126, 172

Balsamo A. 162

Balsan S, 175

Bandivdekar A, 93

Barbe P, 34

Barile M, 98

Barkey R, 115

Barnes N, 31

Baroncelli G, 177

Barral V, 62

Bartolome R, 172

Baumann J, 52, 145

Beaufrere B, 148

Bechinger D, 66

Belgorosky A, 29, 105

Benahmed M. 84

Benderly A, 140, 176

Benz R, 15

Bergada C. 105

Berger B, 94

Bernasconi S, 123,146, 147, 168, 170

Berry J, 138

Bertelloni S, 177

Bertrand J, 83, 85

Besser G, 31, 61, 73

Betts P, 138, 166

Betuel H, 132

Betuel J, 45

Bey-Omar F, 148
Beyer P, 95

Biasco $G, 162$

Bick T, 14

Bidlingmaier $F, 4,26,36,155$

Bierich J, 78

Binoux M, 87

Blum W, 78

Blumberg A, 118

Boerger D, 144

Bollati A, 99

Bolme P, 16

Bonacini M, 170

Bonatesta $\mathrm{P}, 102$

Bonaventure J, 81

Bordigoni P, 160

Boselli E, 168

Bosson D, 3, 18

Bougneres P, 48, 165

Bourguignon $\mathrm{J}, 81$

Bourguignon JP, 6

Bozzola M, 23, 67

Braemswig J, 113

Brambilla P, 48

Brandi A, 38

Bratta P, 102

Brauner R, 47, 62, 70, 175

Bression D, 38

Brock-Jacobsen, 92

Broessler C, 136

Brook C, 10, 21, 53, 75, 117

Brunelles F, 163

Burman K, 98

Bustillo M, 7I

Butler G, 116

Buus O, 139

Buzi F, 60

Cabrol S, 153

Cacciari E, 58, 69, 125, 161, 162

Cadillon E, 28

Candidi F, 58

Canlorbe P, 33

Cantu M, 123

Carrascosa A, 2, 126

Casanova $\mathrm{M}, 33$

Cassio A, 162

Castano L, 165

Catbeh N, 148

Cavallo L, 102

Cavitte J, 120

Cesari C, 125

Challa A, 180

Chantler C, 158

Chapman T, 24

Charlesworth M, 61

Chatelain P, 45, 80, 83, 85148

Chatziliami A, 159

Chaussain J, 5, 48, 150, 165

Chauvin M, 84

Chiari G, 170

Chiesa M, 109, 179

Chiumello G, 99

Chrzanowska B, 149

Cicognani A, 161

Cisternino M, 67

Claustres M, 80

Clayton P, 22

Colaco M, 93

Colle M, 74

Colli C, 58
Collu R, 7

Commentz J, 19

Corvol M, 81, 82

Coude F, 72

Couillin PH, 139

Craen M, 3

Cravidi C, 123

Crosnier H, 70

Cuatrecasas J, 2

Cursted T, 137

Czernichow P, 40, 50, 163, 169

Dacou-Vout, 135

Daffos F, 87

Dahlberg P, 104

Dall'Agnola A, 109, 179

Damkjaer M, 151

Damkjaer N, 139

David M, 45, 127

David M, 132

Davies K, 42

Davies P, 73

Dayer-Metroz M, 20

De Amici M, 23

De La Tour B, 8

De Luca F, 101

De Muinck-Keizer, 30

De Panizza G, 99

De Papendieck, 105

De Peretti E, 28, 84

De Stefano P, 67

Debougnoux G, 6

Degenhart H, 30

Delemarre H, 39, 89

Desai M, 93

Devesa J, 71

Dickerman Z, 115

Dimartino-Nardi, 142

Doehlemann C, 36

Doerner G, 91

Doerr H, 4, 26, 36

Donati S, 69

Donnadieu M, 38, 63

Dousset B, 67, 160

Drayer N, 1, 24

Drop S, 30

Drucker S, 134

Du Caju M, 3, 79

Ducharme J, 7

Dumic M, 134

Dumontier M, 82

Dunger D, 59

Dwyer D, 98

Dyas J, 25

Eberle A, 52, 145

Eisenmenger W, 4

El Kazen N, 18

Ellwood-Russell, 171

Enberg G, 77

Erb TH, 52

Escofet A, 172

Evain-Brion D, 38, 63, 65

Evans B, 128

Facchinetti F, 147

Faiman G, 51

Fainmesser P, 51

Fasano A, 100

Fehres I, 76
Feldt-Rasmussen, 92

Fellous M, 33

Ferrara A, 100

Flexer Z, 51

Flodh H, 2

Florell K, 77

Forabosco A, 161

Forest M, 9, 127

Forestier F, 87

Franchimont P, 6

Franckson J, 18

Francois R, 148

Frederich F, 83

Frejaville E, 125

Frisch $\mathrm{H}, 54$

Gahnem F, 115

Galin J, 7

Garabedian M, 175

Garnier P, 38, 63, 65

Gasparini N, 100

Gauthier TH, 34

Gelander L, 68

Genazzani A, 147

Gepts W, 163

Gerard A, 6

Gerhard I,13

Gerzer R, 36

Ghizzoni L, 123, 147, 168

Gibb W, 7

Gibson B, 12

Gilli G, 13

Giovanelli G, 146, 168

Girard F, 141, 153

Girard J, 52, 118, 145

Glorieux F, 42

Goecke B, 129

Goetz F, 91

Goetz J, 132

Gourmelen M, 153

Grant D, 133

Greene S, 158

Grimberg R, 175

Griva E, 180

Grossman A, 61

Grueters A, 11, 94, 95

Guandalini S, 100

Guillot M, 169

Gupta D, 129, 146

Gusine M, 2

Gustafsson J, 137

Gutekunst R, 106

Haack D, 26

Hadziselimovic, 118

Hagenfeldt L, 137

Haglund B, 131

Hall $\mathrm{K}, 77,86$

Hanna C, 103

Hansson U, 86

Hardouin S, 87

Hartmann K, 76

Hartmann P, 66

Hartmann W, 43

Haschke F, 174

Hasenpusch $U, 106$

Hauffa B, 27

Hauptmann G, 132

Hazebroek F, 30

Heidemann P, 8, 11, 156 
Heinrich U, 37, 76, 96

Heinze E, 66, 90

Heit W, 43

Helge $\mathrm{H}, 11,94$

Hellerstroem C, 40

Hellwege $\mathrm{H}, 19$

Henkel R, 19

Henrichs I, 15

Herkner K, 56

Hernandez M, 63

Hindmarsh P, 10

Hinkel K, 114

Hitzler $\mathrm{H}, 43$

Hochberg Z, 14, 140, 176

Holl R, 66

Holownia P, 117

Honour J, 117

Horak B, 136

Hughes I, 25, 31, 128

Illig R, 44, 111

Iorcansky S, 105

Jaffe M, 176

Jerzenbeck E, 113

Job J, 33, 63, 65, 150

Jones J, 60, 158

Josefsberg Z, 64

Joss E, 112

Kahana L, 140

Karjalainen J, 49

Karlsson F, 104

Karp M, 51

Karpe B, 16

Kauli R, 119

Keller U, 152

Kelnar C, 116

Keret R, 64, 119

Ketelslegers J, 41

Kina S, 18

Kinarti H, 64

Kleij M, 144

Klett M, 96

Klinga K, 13

Knip M, 49

Knorr D, 4, 26, 36, 152

Knudtzon J, 110

Kohnert K, 164

Konitsiotou D, 135

Kontiainen S, 97

Koskimies A, 35

Kraan G, 24

Krabbe S, 124, 151

Kraus C, 156

Kreuter R, 46

Krueger D, 146

Kuhnle U. 152

Kuroda Y, 178

L'Allemand D, 11, 94, 95

La Neve A, 102

Lafranchi S, 103

Lahlou N, 5, 120

Lallemand D, 62

Lang R, 36, 37

Lapatsanis P, 180

Laron Z, 64, 51, 115, 119

Larsson A, 137

Lassarre C, 87

Lassrich A, 144

Laurence K, 25

Lautenschlager, 97

Lechner B, 78

Lecoq A, 127

Lee $D, 166$

Lefebvre J, 169

Leheup B, 67, 160
Leiberman E, 154

Lemerle J, 150

Leppink G, 107

Leroy $\mathrm{P}, 33$

Levy-Marchal C, 169

Liapi C, 38, 65

Lichtwald K, 136

Lightman S, 59

Lima L, 71

Limal J, 167

Lindner $\mathrm{CH}, 88$

Lischka A, 56

Lombes A, 163

Lopez-Cuevas I, 108

Lowe D, 31

Lundin $\mathrm{G}, 86$

Luthman $H, 131$

Maccario R, 23

Mackenzie I, 17

Maenpaa J, 97

Maes M, 41

Mahadik C, 93

Maiolino M, 101

Malvaux P, 3

Mandel S, 103

Mandini M, 69

Mappus E, 28

Marcellini C, 147

Marchal CL, 50

Marcus C, 16

Mari S, 147

Marti C, 20

Mattila A, 35

Mazzanti L, 161

Mazzetti M, 123

McCaughey E, 166

Mehls O, 13

Mengreli $\mathrm{CH}, 159$

Menzel D, 27

Mialot J, 120

Mialot M, 120

Miyao M, 178

Moeller H, 129

Momdain-Monval, 120

Morel Y, 9, 45, 132, 148

Morera A, 84

Moretta A, 23

Morre M, 74

Moschetini V, 58

Moses S, 154

Mueller K, 129

Mueller M, 96

Mueller-Wiefel, 13

Mugnier E, 47

Muritano MR, 111, 130

Mustonen A, 49

Nahoul K, 120

Natali G, 58

Naville D, 83

New M, 134, 142

Ngafoong L, 73

Nihoul-Fekete, 163

Nuzzi G, 170

Nyberg M, 97

O'Connell A, 142

O'Riordan J, 42

Oefverholm U, 86

Ohkawa T, 91

Olive D, 67,160

Pagni G, 125

Pampalone A, 99

Pantelakis S, 159

Papadakou-Lag, 159

Papasteriadou, 135
Parisini O, 161

Partesotti S, 125, 162

Partsch C, 8, 17

Perason D, 12

Peillon F, 38

Pellacani A, 161

Perez J, 45

Perheentupa J, 35, 157

Perlman R, 14

Perrard-Sapori, 83, 85

Persson B, 86

Pesonen K, 35

Petersen K, 139

Pierret T, 141

Pierson M, 67, 160

Pifferi M, 125

Piot A, 5

Pirazzoli P, 69

Polimeni A, 101

Pollak A, 56

Pombo M, 71

Potau N, 2, 172

Pouplard-Bart, 167

Povoa G, 86

Prader A, 44, 130, 143

Prager-Lewin R, 115

Preece M, 55, 60, 73, 158

Prevot C, 47, 70, 82, 175

Price D, 22, 57

Pringle P, 10, 21, 53

Putet G, 173

Radeke H, 156

Ranke M, 78

Ransley P, 31

Rapaport M, 51

Rappaport R, 47, 62, 70, 175

Rascher W, 37

Raux-Demay M, 141

Read A, 42

Read G, 32

Reed W, 98

Rees L, 73, 158

Rege C, 93

Reggiani A, 162

Renier G, 7

Reznik Y, 121

Rigo J, 173

Rime J, 118

Ritzen E, 131

Rivarola M, 29, 105

Rivest $\mathrm{R}, 46$

Rochiccioli P, 34, 72

Rodda C, 73

Roger M, 5, 45, 120, 165

Rohde W, 91

Rolland De Ravel, 28

Romer T, 55, 149

Rondanini, 99

Rooman R, 79

Ross R, 61, 73

Roy M, 47

Ruedas E, 74

Ruffini A, 69

Ruitton A, 83, 85

Rumsby G, 133

Sack J, 98

Saez J, 83, 85

Saggese G, 177

Salardi S, 162

Salle B, 173

Sandomenico M, 100

Sandri S, 161

Sangiovanni M, 69

Santi V, 125

Santiago J, 48

Santoro L, 69

Sassolas G, 148

Satour P, 167

Saudubray JM, 163

Savage M, 31, 61, 73

Schaerer K, 13

Schafmayer A, 156

Schally A, 5, 64, 120

Schatten C, 174

Schellong G, 113

Scherrer H, 38

Schilling, 174

Schimpff R, 23

Schlickenrieder, 43

Schlueter H, 11

Schmid M, 174

Schober E, 54

Schoenberg D, 13, 76, 96, 136

Schoenle E, 111

Schrander-ST, 89

Schwarz HP, 112, 122

Scott-Morgan L, 166

Seboun E, 33

Seckl J, 59

Sempe M, 45

Senterre J, 173

Severi F, 67

Shalet $S, 12,22,32,57$

Sheth A, 93

Shirodkar V, 93

Sinnecker G, 88

Sippell W, 8, 17, 26

Siracusano M, 101

Sizonenko P, 20, 46, 111, 121

Smith P, 10, 53, 75

Smolarek R, 106

Sorgo W, 66

Spano C, 58

Spanos E, 135

Specchio L, 102

Stahl F, 91

Stangenberg M, 86

Stanhope R, 21, 117

Stegner E, 19

Stoner E, 142

Strindberg B, 2

Strom T, 4, 36, 155

Stubbe P, 11, 106

Sultan $\mathrm{CH}, 80$

Surtees R, 57

Svensson E, 137

Swift P, 31, 171

Swindell R, 12

Szoke B, 64

Taborda A, 71

Tagliaro F, 109, 179

Takeda E, 178

Tamanini A, 109, 179

Tang Z, 20

Tassinari D, 16

Tassoni P, 58

Tato L, 67, 109, 179

Tauber J, 34

Tauber M, 34, 72

Tellarini M, 58

Teller W, 15, 43, 66

Tenore A, 100 
Tsatsoulis A, 32

Tsirka A, 180

Tubiana N, 153

Tubiana-Rufi N, 169

Tulassay T, 37

Uboldi F, 34, 72

V. Willebrand, 157

Valtorta A, 23

Van Kalsbeek E, 107

Van Vliet G, 3

Van Wijk-Hoek, 107

Vanderschueren, 3

Vanelli M, 123, 168, 170

Vas F, 93

Vecsei P, 136
Veldkamp E, 39

Ventura M, 101

Venturini I, 170

Venturoli V, 161

Veter U, 43, 90

Vicens-Calvet, 2, 172

Viinikka L, 35

Virdis R, 123, 168, 170

Visser H, 30

Vittorangeli R, 168

Volta C, 123

Volta U, 162

Von Doebeln U, 137

Vrionis C, 180

Waldhauser F, 54
Wartofsky L, 98

Wass J, 61

Weber B, 11, 95

Weil J, 36, 155

Weisman Y, 176

Werder E, 44

Wilkin T, 166

Willeput J, 82

Willi U, 143

Willig R, 19, 88, 144

Wilton P, 104

Wit J, 107

Withehead E, 32

Woloszczuk W, 174

Wolthers B, 24

Wood P, 138
Wu F, 116

Yeste D, 126

Yi-Yan Y, 60

Zachmann M, 44, 108, 130, 143

Zadik Z, 140

Zamboni G, 109, 179

Zapf J, 43

Zilberstein D, 98

Zucchini M, 58

Zucchini S, 162

Zumsteg U, 52, 118

Zuppinger K, 112, 122

Zuppinger U, 52

Zurbruegg R, 118 